This item was submitted to Loughborough's Research Repository by the author.

Items in Figshare are protected by copyright, with all rights reserved, unless otherwise indicated.

\title{
Actuator dynamics augmented DOBC for a small fixed wing UAV
}

PLEASE CITE THE PUBLISHED VERSION

https://doi.org/10.1109/ICIT.2018.8352149

PUBLISHER

(c) IEEE

VERSION

AM (Accepted Manuscript)

LICENCE

CC BY-NC-ND 4.0

REPOSITORY RECORD

Smith, Jean, Jun Yang, Cunjia Liu, and Wen-Hua Chen. 2018. "Actuator Dynamics Augmented DOBC for a Small Fixed Wing UAV". figshare. https://hdl.handle.net/2134/28298. 


\title{
Actuator Dynamics Augmented DOBC for A Small Fixed Wing UAV
}

\author{
Jean Smith, Jun Yang, Cunjia Liu and Wen-Hua Chen \\ Department of Aeronautical and Automotive Engineering \\ Loughborough University \\ Leicestershire, LE11 3TU, United Kingdom \\ \{J.Smith5, J.Yang3, C.Liu5, W.Chen\}@lboro.ac.uk
}

\begin{abstract}
This paper presents an actuator dynamics augmentation of the classical Disturbance Observer Based Control (DOBC) approach for control of a small fixed wing Unmanned Aerial Vehcile (UAV). The proposed method modifies the observer to include actuator dynamics. This augmentation allows for significantly higher observer gains in practice than traditional DOBC in the presence of actuator dynamics, resulting in better disturbance rejection performance. The actuator states are unmeasurable, so are also estimated by the proposed observer using an actuator model and the aircraft state. The actuator modelling process of the UAV is provided in detail. The closedloop stability as well as observer tuning guidelines are discussed. The performance improvement is demonstrated first in numerical simulation and validated with flight test results using a small UAV.
\end{abstract}

Index Terms-actuator dynamics, disturbance rejection, disturbance-observer-based control, unmanned aerial vehicles

\section{INTRODUCTION}

Small-scale Unmanned Aerial Vehciles (UAVs) have gained extensive applications in various industrial sectors, such as intelligent traffic, remote sensing and search and rescue [1]. The small-scale fixed wing UAV under consideration is operated outdoors. The outdoor environment will impose various external disturbances on the aircraft. These unpredictable disturbances usually result in undesirable flying behaviour, with small UAVs being affected more than larger aircraft [2]. Consequently, one of the most crucial tasks of flight control for UAVs is to enhance disturbance rejection ability.

Various elegant advanced control approaches have been proposed for disturbance rejection flight control of smallscale UAVs (see [3]-[6] and the references therein). In [3] a second-order sliding mode control approach, called super twisting control, has been utilized for the attitude tracking of a quadrotor. An optimal control method with anti-windup augmentation using linear quadratic control has been proposed in [4] for autonomous soaring applications. Zhao et al. proposed a nonlinear robust adaptive control approach using immersion and invariance technique for tracking and disturbance rejection of quadrotors [5]. In [6], the combination of integral model predictive control and nonlinear $H_{\infty}$ control

This work is supported in part by the UK Engineering and Physical Science Research Council (EPSRC) under the Grants EP/P012868/1 \& EP/R005494/1. has been developed for robustness and disturbance rejection performance enhancement of quadrotors.

Besides the above various advanced control approaches, Disturbance Observer Based Control (DOBC) methods recently have been developed to address the disturbance rejection problem in UAVs [7]-[9]. Since the external disturbances are directly estimated via a disturbance observer, it is possible to counteract the undesirable influence caused by disturbances in UAVs in a timely manner [8]. As such, it has been reported that the DOBC approach exhibit outstanding disturbance rejection performance as compared to other feedback-based control approaches [10].

In spite of the extensive development of advanced disturbance rejection control approaches, very few have explicitly taken into account the actuator dynamics within the closedloop system design and analysis. Although the actuator dynamics of UAVs are generally much faster than kinematics and attitude dynamics, there is generally a tangible inertia in their dynamics, as well as potential time lag between demand and action. The dynamic profile of a typical physical actuator is shown by Fig. 1. The characteristics of the response would vary depending on the system under consideration.

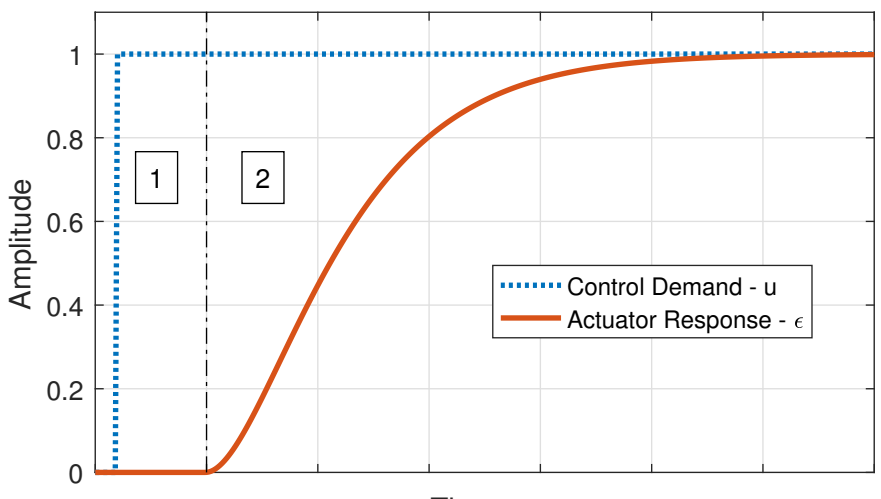

Time $\rightarrow$

Fig. 1: A generalised actuator response to input step. Stage 1 indicates response delay, while stage 2 is the dynamic response. The duration of each stage varies significantly between actuators.

While $u$ is the demanded control action, $\epsilon$ is the applied control action which affects the system response. The classical disturbance observer takes $u$ as its input, assuming $u \approx \epsilon$, 
as direct measurement of actuator states is not commonly available. This assumption is generally sufficient for good performance. However when the observer dynamics become faster (more sensitive), the assumption is no longer sufficient; the mismatch between $u$ and $\epsilon$ may be falsely estimated as disturbances on the system. This assumption therefore essentially constrains the classical observer dynamics to a rate low enough that $u \approx \epsilon$. For good disturbance rejection of unpredictable, non-steady disturbances, it is required that the observer dynamics be faster than that of the disturbance [11]. This is the case for small UAVs subjected to non-continuous atmospheric disturbances. In this work we present an actuator augmentation to the classical disturbance observer, which is referred to as Actuator augmented Disturbance Observer Based Control (ADOBC) within this discussion for clarity. This adds actuator dynamics to the observer, meaning it is no longer assumed that $u \approx \epsilon$. This allows for faster observer dynamics, resulting in improved disturbance rejection.

It is pertinent to remember that small UAVs actuators have fast dynamics. This means the performance benefit demonstrated herein is likely to be more noticeable for systems with slower actuators, such as large aircraft or industrial processes.

Furthermore, as the actuator states on a small UAV are not typically available as a measurement, an additional state observer which utilises an actuator model is developed to reconstruct the actuator states. This enabled flight testing of the method on a target platform, a Skywalker X8 UAV shown in Fig. 2.

\section{System AND ACtUATOR Modelling}

\section{A. Aircraft Model}

In this work, we considered that lateral channel of the aircraft dynamics as this is generally more sensitive to atmospheric disturbances. For control design and numerical simulation, the following linear state space model was used

$$
\underbrace{\left[\begin{array}{c}
\dot{p} \\
\dot{\phi}
\end{array}\right]}_{\dot{\boldsymbol{x}}}=\underbrace{\left[\begin{array}{cc}
-27.5 & 0 \\
1 & 0
\end{array}\right]}_{\boldsymbol{A}} \underbrace{\left[\begin{array}{c}
p \\
\phi
\end{array}\right]}_{\boldsymbol{x}}+\underbrace{\left[\begin{array}{c}
224 \\
0
\end{array}\right]}_{\boldsymbol{B}} u,
$$

where $p$ is the roll rate, $\phi$ is the roll angle and $u$ is the system input; the roll elevon control demand. The state output being controlled, $y$, is $\phi$, which defines $\boldsymbol{C}=\left[\begin{array}{ll}0 & 1\end{array}\right] . D$ is taken to be 0 . The numerical values for the state and control matrices were obtained from system identification of the Skywalker X8.

\section{B. Actuator Models}

To represent the aircraft actuators, two different models were used,

$$
G_{f}(s)=\frac{1}{\tau s+1}, \quad G_{s}(s)=\frac{\omega_{n}^{2}}{s^{2}+2 \zeta \omega_{n} s+\omega_{n}^{2}},
$$

where $G_{f}(s)$ and $G_{s}(s)$ are the first and second order actuator transfer functions respectively, $\tau$ is the time constant, $\omega_{n}$ is the natural frequency and $\zeta$ is the damping ratio.

Generally, it would be expected that the second order model better represents the actuator dynamics of the aircraft. For

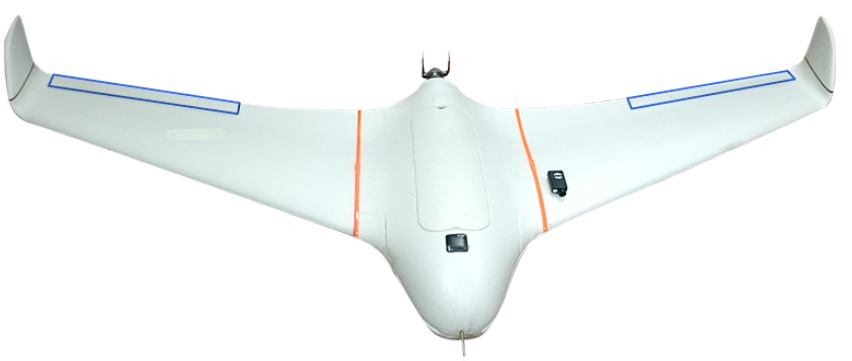

Fig. 2: The Skywalker X8 used in this work, with the control surfaces highlighted in blue. Aircraft wingspan $2.12 \mathrm{~m}$, weight $3 \mathrm{~kg}$.

this reason, the second order model is used in the numerical simulation of aircraft dynamics and the first order model is used in observer design. Using the first order model in the observer ensures that some modelling error will exist, which the method is expected to account for. This also demonstrates performance in the absence of an ideal actuator model, which is unlikely to exist in many applications.

\section{Test Methodology}

To obtain the actuator model parameters, VICON motion tracking was used to record actuator deflection rates with the actuators attached to the aircraft in its flight configuration. To accurately assess the response, it was required to synchronise the control demand signal with the measured actuator position. Using a Robotic Operating System (ROS) network and Pixhawk PX4, it was possible to record the actuator demand and true deflection in the same environment, simultaneously. This enabled accurate measurement of both response delay and actuator dynamics, as depicted in Fig. 1. Using ROS to collect the data relies on a data network to transmit messages from the various sources. Delay within the network was measured to be of an order below $1 \mathrm{~ms}$, which was well within our margin of error and so would not affect the results.

\section{Actuator Dynamics Modelling}

For response modelling, five full deflection steps were recorded. In all tests, a response delay of $0.08 \mathrm{~s}$ was recorded. The mean dynamic response of the recorded steps can be seen in Fig. 3.

Within this response, two key features exist. Firstly, we notice the inertial component, with a clear acceleration and deceleration present at the beginning and end of the step. These two periods are linked by a constant velocity component. This is expected, as the servo actuators tested feature an internal control scheme which regulates this response. Although it is possible to replicate this behaviour when modelling the actuators by saturating the velocity to the appropriate value, it is non-trivial to include such a model in the control design. Instead, the first and second order models were tuned to produce as similar a response as possible for control design. Fig. 3 also contains the responses of a first and second order actuator model tuned to match the response.

For the simulation and control design models, the responses were tuned to be slower than that indicated in Fig. 3 to account 




Fig. 3: The mean VICON response compared to the first and second order models used to represent the response, with response delay removed.

for the measured $0.08 \mathrm{~s}$ time delay. This was done as the delay would be an inevitable part of flight testing, so using models which accounted for this would better represent the final system, offering improved performance.

\section{ACtuator-Dynamics Augmented DOBC}

To include the actuator model acquired in Section II, the DOBC scheme used previously must be modified and expanded. Previous DOBC design in [9] has used a state space model in the form

$$
\dot{\boldsymbol{x}}=\boldsymbol{A} \boldsymbol{x}+\boldsymbol{B} u+\boldsymbol{d}_{l x},
$$

based on the aircraft model given in (1), where $\boldsymbol{A}$ and $\boldsymbol{B}$ are the matrices for aircraft dynamics and control effectiveness, $\boldsymbol{x}$ is the system state vector, $u$ is the input command and $\boldsymbol{d}_{l x}$ are the lumped disturbances acting on the system. This model does not take into account any actuator dynamics, assuming that the demanded control $u$ is the applied control. This is acceptable for observer dynamics which are slower than the actuator dynamics. However, for good DOBC function when a non-steady disturbance is considered, it is practically required that the observer dynamics are faster than that of the disturbance. For flight control, wind gust disturbances have fast dynamics, meaning a faster observer is needed. Consequently, the observer dynamics can approach the point where any difference between the demanded control and true actuator positions can be detected as a disturbance on the system. In an attempt to deal with this, we adapt a previous DOBC method for helicopter flapping angles [7], to include a model of the actuator dynamics in the observer. The new model then becomes the cascaded system shown below

$$
\begin{gathered}
\dot{\boldsymbol{x}}=\boldsymbol{A}_{1} \boldsymbol{x}+\boldsymbol{B}_{1} \epsilon+\boldsymbol{d}_{l x}, \\
\dot{\epsilon}=A_{2} \epsilon+B_{2} u,
\end{gathered}
$$

where $\boldsymbol{A}_{1}=\boldsymbol{A}, \boldsymbol{B}_{1}=\boldsymbol{B}$ and $\epsilon$ is the actuator deflection based on the demanded control input $u, A_{2}$ and $B_{2}$ are the state space representations of the first order actuator model in (2). By moving the control input $u$ to be an input to the actuator position model rather than directly in the system state, we are able to include the actuator modelling into the system. This model is however limited. As shown in Fig. 3, neither the first or second order actuator model are able to represent the actuator dynamics with true accuracy. By using the DOBC technique, the actuator model estimate $\epsilon$ can be improved by including an estimation term based on state measurements.

\section{A. Actuator/Disturbance Observers Co-Design}

First, we take the actuator model from (4) to define the dynamics component of our system

$$
\dot{\epsilon}=A_{2} \epsilon+B_{2} u,
$$

and rearrange the system state equation from (4) to define our measurement component

$$
\boldsymbol{B}_{1} \epsilon=\dot{\boldsymbol{x}}-\boldsymbol{A}_{1} \boldsymbol{x}-\hat{\boldsymbol{d}}_{l x},
$$

where $\boldsymbol{d}_{l x}$ has been replaced with its estimate $\hat{\boldsymbol{d}}_{l x}$, assuming it will be defined later. We can then define an estimator for the actuator position

$$
\dot{\hat{\epsilon}}=A_{2} \hat{\epsilon}+B_{2} u+\boldsymbol{L}_{\epsilon}\left(\dot{\boldsymbol{x}}-\boldsymbol{A}_{1} \boldsymbol{x}-\hat{\boldsymbol{d}}_{l x}-\boldsymbol{B}_{1} \hat{\epsilon}\right),
$$

where $\hat{\epsilon}$ is the estimated actuator position and $\boldsymbol{L}_{\epsilon}$ is the estimator gain parameter. However, in its current form, this estimator features a measurement which is unavailable, $\dot{\boldsymbol{x}}$. We continue with the standard DOBC method of defining an auxiliary equation to remove $\dot{\boldsymbol{x}}$. First, we define

$$
\hat{\epsilon}=z_{2}+\boldsymbol{L}_{\epsilon} \boldsymbol{x}
$$

which also gives

$$
\dot{\hat{\epsilon}}=\dot{z}_{2}+\boldsymbol{L}_{\epsilon} \dot{\boldsymbol{x}} .
$$

By substituting $\dot{\hat{\epsilon}}$ from (9) into (7), we get

$$
\dot{z}_{2}+\boldsymbol{L}_{\epsilon} \dot{\boldsymbol{x}}=A_{2} \hat{\epsilon}+B_{2} u+\boldsymbol{L}_{\epsilon}\left(\dot{\boldsymbol{x}}-\boldsymbol{A}_{1} \boldsymbol{x}-\hat{\boldsymbol{d}}_{l x}-\boldsymbol{B}_{1} \hat{\epsilon}\right),
$$

which allows for removal of the $\boldsymbol{L}_{\epsilon} \dot{\boldsymbol{x}}$ term which appears in both sides of the equation. Finally, we can define the disturbance observer with actuator dynamics by combining (10) and (8) into

$$
\left\{\begin{array}{l}
\dot{z}_{2}=A_{2} \hat{\epsilon}+B_{2} u+\boldsymbol{L}_{\epsilon}\left(-\boldsymbol{A}_{1} \boldsymbol{x}-\boldsymbol{B}_{1} \hat{\epsilon}-\hat{\boldsymbol{d}}_{l x}\right) \\
\hat{\epsilon}=z_{2}+\boldsymbol{L}_{\epsilon} \boldsymbol{x} .
\end{array}\right.
$$

This has a similar form to the basic disturbance observer designed previously, except for featuring dynamics as well as a measurement component. This does mean that the process of tuning the observer gain $\boldsymbol{L}_{\epsilon}$ is more complex than for the basic observer. For the basic observer, the $\boldsymbol{L}$ gain in essence controls the convergence rate of the estimate to the true disturbance; generally, a high observer gain is desirable. For the actuator observer, the $\boldsymbol{L}_{\epsilon}$ gain not only controls the convergence rate but also changes how $\hat{\epsilon}$ is generated. This is discussed in detail in Section III-C. 
The disturbance observer design for lumped disturbances on the UAV states is similar to [9]. However, note that the state $\epsilon$ is not available for disturbance observer design now. Instead, we should utilize its estimate $\hat{\epsilon}$ generated by (11) for disturbance observer design. The new disturbance observer is then designed as

$$
\left\{\begin{array}{l}
\dot{\boldsymbol{z}}_{1}=-\boldsymbol{L}\left(\boldsymbol{z}_{1}+\boldsymbol{L} \boldsymbol{x}\right)+\boldsymbol{L}\left(-\boldsymbol{A}_{1} \boldsymbol{x}-\boldsymbol{B}_{1} \hat{\epsilon}\right) \\
\hat{\boldsymbol{d}}_{l x}=\boldsymbol{z}_{1}+\boldsymbol{L} \boldsymbol{x} .
\end{array}\right.
$$

Define the observation errors as $e_{\epsilon}=\epsilon-\hat{\epsilon}$ and $e_{d}=\boldsymbol{d}_{l x}-$ $\hat{\boldsymbol{d}}_{l x}$. Combining the system dynamics (4) and the observers (11) and (12), the error dynamics of the observers are governed by

$$
\left[\begin{array}{c}
\dot{e}_{\epsilon} \\
\dot{\boldsymbol{e}}_{d}
\end{array}\right]=\left[\begin{array}{cc}
A_{2}-\boldsymbol{L}_{\epsilon} \boldsymbol{B}_{1} & -\boldsymbol{L}_{\epsilon} \\
-\boldsymbol{L} \boldsymbol{B}_{1} & -\boldsymbol{L}
\end{array}\right]\left[\begin{array}{c}
e_{\epsilon} \\
\boldsymbol{e}_{d}
\end{array}\right]
$$

where we assume that the disturbance is constant. The exponential stability of the observer error dynamics is guaranteed with appropriate design of observer gains $\boldsymbol{L}$ and $\boldsymbol{L}_{\epsilon}$ such that the matrix

$$
\tilde{\boldsymbol{A}}=\left[\begin{array}{cc}
A_{2}-\boldsymbol{L}_{\epsilon} \boldsymbol{B}_{1} & -\boldsymbol{L}_{\epsilon} \\
-\boldsymbol{L} \boldsymbol{B}_{1} & -\boldsymbol{L}
\end{array}\right]
$$

is chosen to be Hurwitz stable.

\section{B. Actuator-Dynamics-Augmented DOBC}

Letting $\boldsymbol{\eta}=\left[\boldsymbol{x}^{T}, \epsilon^{T}\right]^{T}$, the augmented dynamic system is given by

$$
\dot{\boldsymbol{\eta}}=\overline{\boldsymbol{A}} \boldsymbol{\eta}+\overline{\boldsymbol{B}}_{u} u+\overline{\boldsymbol{B}}_{d} \boldsymbol{d}_{l x}
$$

with

$$
\overline{\boldsymbol{A}}=\left[\begin{array}{cc}
\boldsymbol{A}_{1} & \boldsymbol{B}_{1} \\
0 & A_{2}
\end{array}\right], \quad \overline{\boldsymbol{B}}_{u}=\left[\begin{array}{c}
0 \\
B_{2}
\end{array}\right], \quad \overline{\boldsymbol{B}}_{d}=\left[\begin{array}{c}
\boldsymbol{I} \\
0
\end{array}\right] .
$$

With the estimations obtained above, we are now ready to define the disturbance compensation gain. First, we give the composite control law as

$$
u=-\boldsymbol{k}_{1} \boldsymbol{x}-k_{2} \hat{\epsilon}+\boldsymbol{k}_{d} \hat{\boldsymbol{d}}_{l x} .
$$

Letting $\boldsymbol{K}_{x}=\left[\boldsymbol{k}_{1}^{T}, k_{2}^{T}\right]^{T}$, the disturbance compensation gain is designed as

$$
\boldsymbol{K}_{d}=-\left[\boldsymbol{C}\left(\overline{\boldsymbol{A}}-\overline{\boldsymbol{B}}_{u} \boldsymbol{K}_{x}\right)^{-1} \overline{\boldsymbol{B}}_{u}\right]^{-1} \boldsymbol{C}\left(\overline{\boldsymbol{A}}-\overline{\boldsymbol{B}}_{u} \boldsymbol{K}_{x}\right)^{-1} .
$$

It can be shown that the external disturbance can be exponentially compensated from the output channel if the control gain $\boldsymbol{K}_{x}$ and observer gains $\boldsymbol{L}$ and $\boldsymbol{L}_{\epsilon}$ are selected such that $\overline{\boldsymbol{A}}-\overline{\boldsymbol{B}}_{u} \boldsymbol{K}_{x}$ and $\tilde{\boldsymbol{A}}$ are Hurwitz stable, following [12].

\section{Disturbance Observer Gain Selection}

The disturbance observer gain is defined as

$$
\boldsymbol{L}_{t}=\left[\begin{array}{c}
\boldsymbol{L} \\
\boldsymbol{L}_{\epsilon}
\end{array}\right]
$$

where $\boldsymbol{L}_{t} \in \mathbb{R}^{3 \times 2}, \boldsymbol{L} \in \mathbb{R}^{2 \times 2}$ and $\boldsymbol{L}_{\epsilon} \in \mathbb{R}^{1 \times 2}$ are the total, state and actuator observer gains, respectively. For the state observer gain $\boldsymbol{L}$, which is common to both the DOBC and $\mathrm{ADOBC}$, it was tuned for best acceptable performance based on DOBC simulations. The same gain was then also set as the baseline $\boldsymbol{L}$ gain for the ADOBC controller. As will be shown in the results, a high $\boldsymbol{L}$ gain which performs well with the DOBC scheme will generally also perform well for the ADOBC scheme; the reverse is not true. This means it is more reasonable to tune the baseline $\boldsymbol{L}$ gain for good DOBC performance. In the results section, this base gain will be multiplied by scalars to show the effect of increasing gains for the two schemes. This is achieved with

$$
\boldsymbol{L}_{t}=L_{k} \boldsymbol{L}_{t}^{B},
$$

where $L_{k} \in \mathbb{R}$ is the scalar used to tune the gain based on $\boldsymbol{L}_{t}^{B}$, the baseline gain, given as

$$
\boldsymbol{L}_{t}^{B}=\left[\begin{array}{cc}
1.5 & 0 \\
0 & 0.75 \\
0.001 & 0
\end{array}\right]
$$

The selection of $\boldsymbol{L}_{\epsilon}$ warrants some discussion in the presence of model uncertainty. The first point to note is that $\hat{\epsilon}$ is updated only on measurements of the roll rate, $p$. This is intuitive as the control surfaces only affect the roll rate of the aircraft, as seen in the model (1). Secondly, it is clear that the $\boldsymbol{L}_{\epsilon}$ gain is comparatively small compared to the state observer gains. This is a more subtle point to consider. In essence, the balance between $\|\boldsymbol{L}\|$ and $\left\|\boldsymbol{L}_{\epsilon}\right\|$ dictates how much the overall system attributes an external disturbance to either $a$ ) an actuator deflection or $b$ ) an external disturbance. For example, as $\boldsymbol{L}_{\epsilon} \rightarrow 0$, the estimate of $\epsilon$ will tend towards the dynamic model output. Conversely, as $\boldsymbol{L}_{\epsilon} \rightarrow \infty$, the estimate of $\epsilon$ will tend toward the measurement from the aircraft state. The issue arises when a disturbance is also acting on the system. In this case, the balance of observer gains can lead to the disturbance being falsely attributed to an actuator deflection; this causes a degradation of performance. An example of this situation is shown in Figs 4 and 5.

For these demonstration simulations, the first order actuator model used in the ADOBC scheme is intentionally slowed to exacerbate the effect for visual clarity. Two gains are compared, $\boldsymbol{L}_{\epsilon}=\left[\begin{array}{ll}0.5 & 0\end{array}\right]$ and $\boldsymbol{L}_{\epsilon}=\left[\begin{array}{ll}0 & 0\end{array}\right]$; these are labelled as $L_{\epsilon}=0.5$ and $L_{\epsilon}=0$ on the figures respectively. First, a large reference step is introduced at $t=0.5 \mathrm{~s}$. By comparing the actuator estimation plots, we see that the higher $\boldsymbol{L}_{\epsilon}$ is much better at updating the actuator position estimate, accounting for the modelling errors. This also leads to slightly better reference tracking performance. A disturbance is then added at $t=3 \mathrm{~s}$. Here, we see the other effect. The higher $\boldsymbol{L}_{\epsilon}$ gain attributes a significant amount of this disturbance to a false actuator position estimation, while the other assumes it is entirely external. Now, despite the small error in actuator position for $\boldsymbol{L}_{\epsilon}=0$, the disturbance rejection performance is significantly improved.

This parameter requires some manual tuning for best performance and it is expected that the ideal balance will shift depending on the system and nature of the disturbances. In our case, using an $L_{\epsilon}=0.001$ gave the best balance between the two extremes. By maintaining the ratio between $\|\boldsymbol{L}\|$ and $\left\|\boldsymbol{L}_{\epsilon}\right\|$, as achieved by (17), the characteristic performance of 


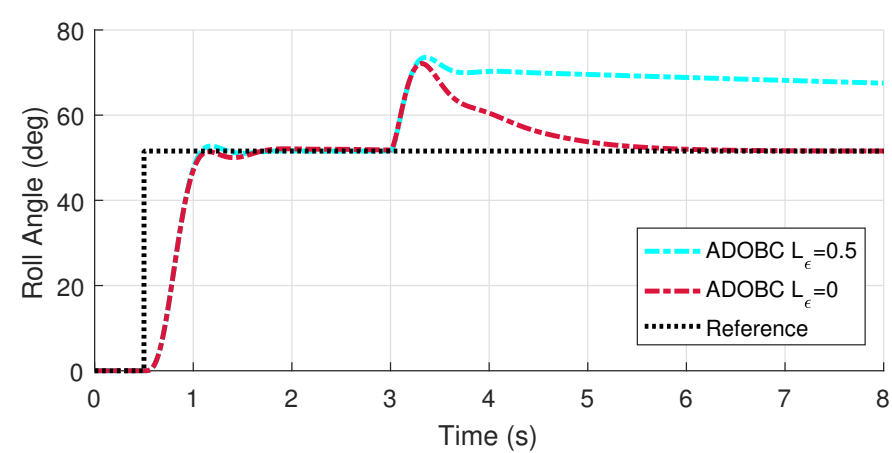

Fig. 4: ADOBC responses with varying $\boldsymbol{L}_{\epsilon}$ gains to a reference step. A disturbance is added at $t=3 s$.
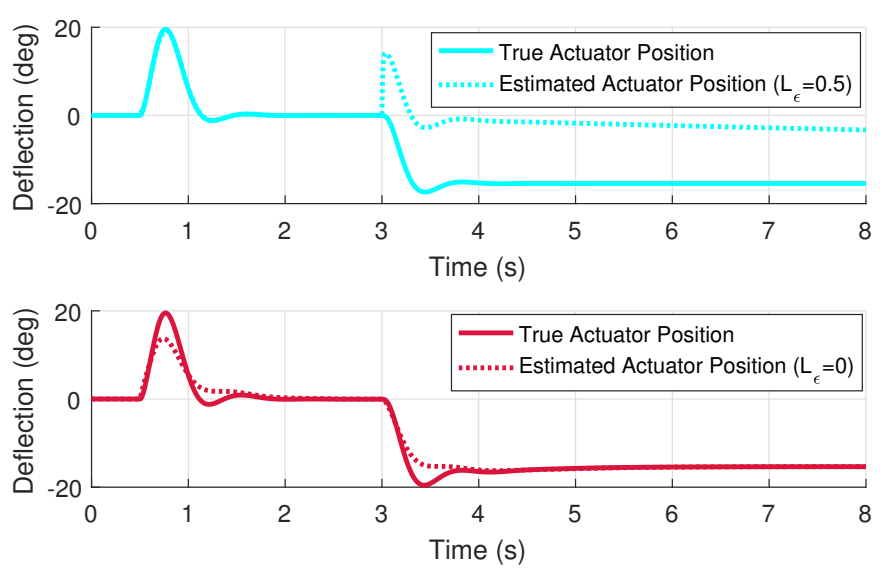

Fig. 5: True and estimated actuator positions for the ADOBC responses with varying $\boldsymbol{L}_{\epsilon}$ gains.

the ADOBC scheme, with respect to this estimation error behaviour, is maintained.

\section{Feedback Control Design}

For this work, a Linear Quadratic Regulator (LQR) was used for baseline feedback control, as in previous work [9]. The LQR tuning was done to provide satisfactory baseline performance. The upper limit of the LQR gains resulted from the actuator dynamics; tuning the LQR for very high response rates induced undesirable effects from the actuators, which led to poor performance. To allow fair assessment of the DOBC techniques, it was paramount to ensure that the $\mathrm{LQR}$ gains were not high enough to excite these dynamics, while still providing good baseline performance.

\section{Simulation AND Experimental Studies}

To demonstrate the advantage of the ADOBC method, performance is first studied in simulation and then validated with flight test results. Within the results, when only the LQR controller is active, the response is denoted as $L Q R$, when the LQR is active and DOBC augmentation is applied, the response is denoted as $D O B C$ and if the $\mathrm{LQR}$ is active and augmented with $\mathrm{ADOBC}$, the response is denoted as $A D O B C$.

\section{A. Numerical Simulations}

Numerical simulations are given to demonstrate the key concepts of the controllers in a fully controlled environment, devoid of any unintentional disturbances. As these results are later supported by flight test results, using a simple linear model for simulation was deemed sufficient to demonstrate the behaviour of the proposed method.

Fig. 6 shows a baseline simulation, comparing LQR, DOBC and $\mathrm{ADOBC}$ performance using the gains in (18). A continuous step disturbance is added at $t=1.5 \mathrm{~s}$ to demonstrate the various responses. Prior to the additional disturbance, the two DOBC schemes perform very similarly to the LQR, as expected. The slight difference being due to actuator dynamics. We notice that the DOBC scheme has slightly more overshoot with the initial reference step. Once the disturbance is added, both DOBC schemes significantly improve performance over the LQR. Here we notice that the ADOBC scheme lags behind the baseline DOBC slightly in disturbance rejection, although with a smoother response.

In Fig. 7 we demonstrate the main benefit of the ADOBC technique. Here, we compare the baseline observers with an $L_{k}=1$ to the same observers with higher gains. Two reference commands are conducted with disturbances added at $t=2 \mathrm{~s}$ and $t=6 \mathrm{~s}$. For the DOBC, the gain was increased by a factor of 3. However, we see that this has introduced oscillations into the response, resulting in far worse performance. Further, the oscillations result from both reference commands and disturbances; this confirms that this is due to the unmodelled actuator dynamics. This observer gain is too high and would need to be reduced for a reasonable response, resulting in slower observer dynamics. A higher gain would only exacerbate the oscillations. To demonstrate the ADOBC benefit, the gain was increased by a far larger factor, chosen as 48 . This is achieved with no degradation in performance, with this response having the best reference tracking and substantially improved disturbance rejection. There is no indication of the oscillations seen in the DOBC response. With such a fast observer, the disturbance rejection rate is now limited by the actuator dynamics, rather than the observer estimation. This shows that the small reduction in disturbance rejection for the ADOBC compared to DOBC (with the same gain), shown in Fig. 6, is easily accounted for by the substantial increase in observer gain which can be applied.

\section{B. Outdoor Flight Test}

For the outdoor test, the control schemes tested within the numerical simulations were compiled to $\mathrm{C}$ code, which ran aboard a Raspberry Pi (RPi) directly connected to a Pixhawk autopilot aboard the test aircraft.

To produce repeatable results, an artificial physical disturbance was introduced. A switch on the transmitter was linked to adding a roll deflection of the elevons of $5^{\circ}$. This was not passed to the control schemes as an input. The resulting aircraft roll would appear as an external step disturbance, allowing for direct and repeatable comparison of disturbance rejection performance. Fig. 8 gives the results. First, it is clear that all DOBC schemes outperform the LQR. Further, it is evident that the DOBC scheme has been tuned to a similar maximum as shown in the numerical simulations, with 


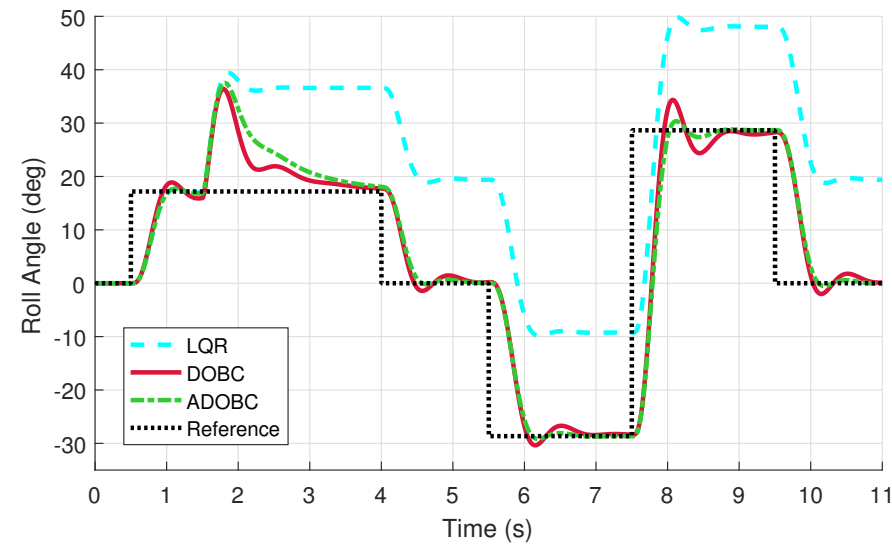

Fig. 6: Comparison of the baseline LQR, DOBC and ADOBC responses to reference commands. A disturbance is added at $t=1.5 \mathrm{~s}$.

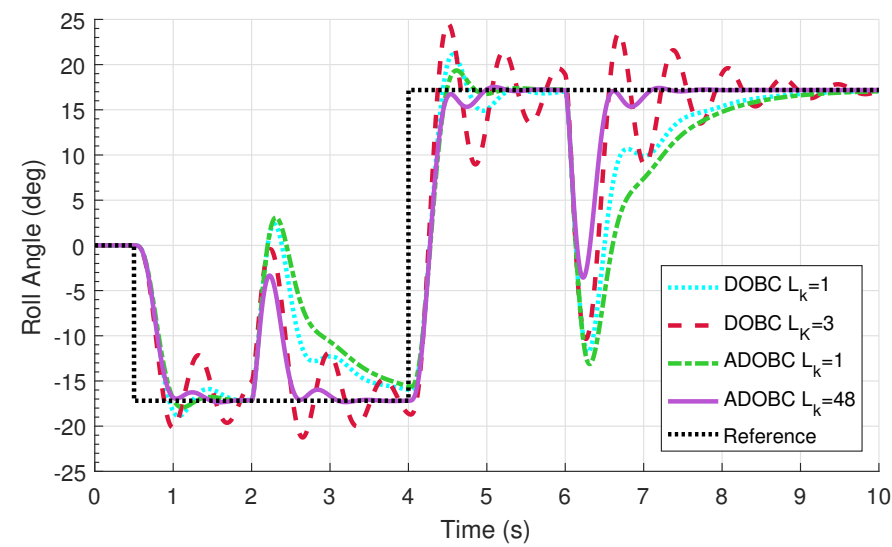

Fig. 7: The effect of increasing observer gains for the DOBC and proposed ADOBC schemes.

oscillations becoming evident. We also see that, as in numerical simulation, the ADOBC scheme lags slightly behind when using equivalent gain. Vitally, we see that with the ADOBC we have been able to substantially increase the observer gain without any oscillation (as seen for the DOBC). This gives smoother performance with better disturbance rejection. This replicates the findings of the simulations. It is also interesting to note that on the $L_{k}=8$ flight, the ADOBC starts at an increased distance from the reference due to an external disturbance. Despite this it is still able to outperform the other schemes with the lowest maximum error. By $t=5 \mathrm{~s}$, all controllers had reached and settled to their steady state condition.

\section{CONCLUSiOnS}

The proposed actuator augmentation of the classical DOBC method has been demonstrated to allow substantial increases in observer gains in practice. This is the case for the majority of physical systems, making this method highly relevant in application. It was shown that the presence of actuator dynamics place limits the applicable classical DOBC gain, where the observer dynamics become fast enough to detect the difference between demanded and applied control. With the proposed

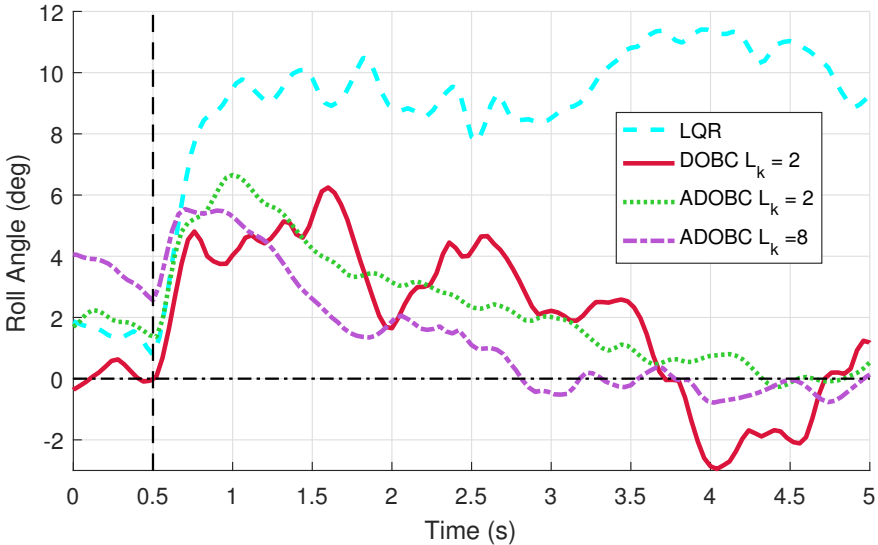

Fig. 8: Flight test data comparing the various control schemes to an identical artificial disturbance added at $t=0.5 \mathrm{~s}$.

ADOBC augmentation, the observer is able to account for this difference, removing this limitation on observer gain. This resulted in substantially improved performance. This was demonstrated with numerical simulations and validated with flight test data. Physical testing demonstrated the benefits of the linear ADOBC on a non-linear physical plant with modelling errors.

\section{REFERENCES}

[1] D. Cabecinhas, C. Rita, and S. Carlos, "A nonlinear quadrotor trajectory tracking controller with disturbance rejection," Control Engineering Practice, vol. 26, pp. 1-10, May 2014.

[2] T. J. Mueller, and J. D. DeLaurier, "Aerodynamics of small vehicles," Annual Review of Fluid Mechanics, vol. 35, no. 1, pp. 89-111, Jan. 2003.

[3] L. Derafa, A. Benallegue, and L. Fridman, "Super twisting control algorithm for the attitude tracking of a four rotors UAV," Journal of the Franklin Institute, vol. 349, no. 2, pp. 685-699, Mar. 2012.

[4] N. E. Kahveci, P. A. Ioannou, and M. D. Mirmirani, "Adaptive LQ control with anti-windup augmentation to optimize UAV performance in autonomous soaring applications," IEEE Transactions on Control Systems Technology, vol. 16, no. 4, pp. 691-707, Jul. 2008.

[5] B. Zhao, B. Xian, Y. Zhang, and X. Zhang, "Nonlinear robust adaptive tracking control of a quadrotor UAV via immersion and invariance methodology," IEEE Transactions on Industrial Electronics, vol. 62, no. 5, pp. 2891-2902, May 2015.

[6] G. V. Raffo, M. G. Ortega, and F. R. Rubio, "An integral predictive/nonlinear H-infinity control structure for a quadrotor helicopter," Automatica, vol. 46, no. 1, pp. 29-39, Jan. 2010.

[7] H. Lu, C. Liu, L. Guo, and W.-H. Chen, "Flight control design for smallscale helicopter using disturbance-observer-based backstepping," Journal of Guidance, Control, and Dynamics, vol. 38, no. 11, pp. 2235-2240, Nov. 2015.

[8] C. Liu, and W.-H. Chen, "Disturbance rejection flight control for small fixed-wing unmanned aerial vehicles," Journal of Guidance, Control, and Dynamics, vol. 39, no. 12, pp. 2804-2813, Dec. 2016.

[9] J. Smith, J. Su, C. Liu, and W.-H. Chen, "Disturbance observer based control with anti-windup applied to a small fixed wing UAV for disturbance rejection," Journal of Intelligent and Robotic Systems, vol. 88, no. 2-4, pp. 329-346, Dec. 2016.

[10] W.-H. Chen, J. Yang, L. Guo, and S. Li, "Disturbance-observer-based control and related methodsAn overview," IEEE Transactions on Industrial Electronics, vol. 63, no. 2, pp. 1083-1095, Feb. 2016.

[11] W.-H Chen, D. Ballance, P. Gawthrop, and J. OReilly, "A non-linear disturbance observer for robotic manipulators," IEEE Transactions on Industrial Electronics, vol. 47, no. 4, pp. 932-938, Aug. 2000.

[12] S. Li, J. Yang, W.-H Chen, X. Chen, "Disturbance observer based control: methods and applications," CRC Press, 2014 